\title{
Dentifrícios fluoretados e o SUS-Brasil: O que precisa ser mudado?
}

\section{Fluoride toothpastes and SUS-Brazil: What needs to be changed?}

\section{Pastas de dientes fluoradas y SUS-Brasil: ¿Qué necesita ser cambiado?}

\author{
Jaime Aparecido Cury ${ }^{1}$ \\ Luis Fernando Bandeira Miranda ${ }^{2}$ \\ Pablo Guilherme Caldarelli ${ }^{3}$ \\ Cinthia Pereira Machado Tabchoury ${ }^{4}$
}

RESUMO: O presente estudo aborda as mudanças necessárias que devem ser implementadas para que toda a população brasileira possa ser beneficiada pelo uso de dentifrícios fluoretados em termos de prevenção de cárie dentária. Foi realizada uma busca da literatura sobre concentração de fluoreto em dentifrícios brasileiros, nas bases de dados Lilacs, PubMed, SciELO e nos arquivos do laboratório de Bioquímica Oral da FOP-UNICAMP. Os resultados mostram que desde o início da década de 1980, quando se iniciou o acompanhamento das concentrações de fluoreto em dentifrícios brasileiros, muitos dentifrícios não são capazes de manter uma concentração mínima de fluoreto solúvel para fornecer benefício anticárie. Este é um problema recorrente não só com os dentifrícios de livre venda presentes no comércio, mas principalmente com aqueles comprados por licitação pública pelo SUS e distribuídos para a população. A regulamentação brasileira vigente (Resolução 79, Anvisa, 28/08/2000) estabelece apenas a concentração máxima de fluoreto total que um dentifrício deve conter, mas não a mínima solúvel necessária para garantir o benefício anticárie da formulação. Uma mudança, estabelecendo a concentração mínima de fluoreto solúvel que um dentifrício deveria manter por determinado tempo após sua fabricação, não só beneficiaria o consumidor brasileiro, mas principalmente todos os usuários do SUS, porque poderia ser referendada nos editais de licitação de compra de dentifrícios.

Palavras Chave: Dentifrícios; Fluoretação;Legislação \&Jurisprudência;Cárie Dentária;Prevenção \&Controle.

1 Faculdade de Odontologia de Piracicaba, Área de Bioquímica e Cariologia

2 Mestrando em Odontologia, Área de Cariologia, Faculdade de Odontologia de Piracicaba, UNICAMP

3 Professor Adjunto de Odontologia em Saúde Coletiva, Universidade Estadual de Londrina, UEL

4 Professora Associada de Bioquímica e Cariologia, Faculdade de Odontologia de Piracicaba, UNICAMP

ISSN 1982-8829 Tempus, actas de saúde colet, Brasília, 14(1), 09-27, mar, 2020. Epub Mai/2020 
ABSTRACT:The present study explores the necessary changes that should be implemented to benefit the entire Brazilian population by using fluoride toothpastes in terms of dental caries prevention. A literature search on fluoride concentration in Brazilian toothpastes was performed in Lilacs, PubMed, and SciELO databases and in the files of the Laboratory of Oral Biochemistry from FOP-UNICAMP.The results show that since the early 1980s, when the monitoring of fluoride concentrations in Brazilian toothpastes began, many toothpastes have not been able to maintain a minimum concentration of soluble fluoride to provide anticaries benefit. This is a recurring problem not only with over-the-counter toothpastes present in the market, but especially with those purchased by public bidding by SUS and distributed to the population. The current Brazilian regulation (Resolution 79, Anvisa, 8/28/2000) establishes only the maximum total fluoride concentration that a toothpaste must contain, but not the minimum soluble fluoride necessary to ensure the anticaries benefit of the formulation. One change, establishing the minimum concentration of soluble fluoride that a toothpaste should maintain for a certain time after its manufacture, would not only benefit the Brazilian consumer, but mainly all SUS users, because it could be countersigned in the toothpaste bidding documents.

Keywords: Dentifrices; Fluoridation;Legislation \&Jurisprudence; Dental Caries;Prevention \&Control.

RESUMEN: El presente estudio aborda los cambios necesarios que deben ser implementados para que toda la población brasileña pueda beneficiarse del uso de dentífricos con fluoruro en términos de prevención de la caries dental. Se realizó una búsqueda en la literatura sobre la concentración de fluoruro en dentífricos brasileños en las bases de datos Lilacs, PubMed, SciELO y en los archivos del laboratorio de Bioquímica Oral FOP-UNICAMP. Los resultados muestran que, desde principios de la década de 1980, cuando empezó el monitoreo de las concentraciones de fluoruro en los dentífricos brasileños, muchos dentífricos no son capaces de mantener una concentración mínima de fluoruro soluble para proporcionar beneficio contra la caries. Este es un problema recurrente no solo con los dentífricos presentes en el comercio, sino especialmente con aquellos comprados por el SUS y distribuidos a la población. La normativa brasileña actual (Resolución 79, Anvisa, 8/28/2000) establece solo la concentración máxima de fluoruro total que un dentífrico debe contener, pero no la mínima soluble necesaria para garantizar el beneficio anti-caries de la formulación. En cambio, estableciendo la concentración mínima de fluoruro soluble que un dentífrico debe mantener durante un cierto tiempo después de su fabricación, no solo beneficiaría al consumidor brasileño, sino que especialmente a todos los usuarios del SUS, ya que, podría ser refrendado en los documentos de licitación de dentífricos.

Palabras clave: Dentífricos. Fluoruración, legislación y jurisprudencia. Caries dental, prevención y control.

\section{INTRODUÇÃO}

Dentifrício fluoretado é considerado o meio mais racional de uso de fluoretos para o controle 
de cárie dentária, pois ao mesmo tempo em que o biofilme dental é removido/desorganizado pela escovação, o fluoreto é liberado na cavidade bucal para reduzir o desenvolvimento de lesões de cárie ("efeito preventivo") ou para ativar a reparação das lesões iniciais já existentes ("efeito terapêutico") ${ }^{1,2}$. O efeito do fluoreto presente nos dentifrícios no controle da cárie dentária está fortemente baseado em evidência de dezenas de estudos clínicos randomizados e controlados ${ }^{3}$, entretanto para que um dentifrício seja eficaz no controle da cárie dental é indispensável que o fluoreto esteja quimicamente solúvel na formulação ${ }^{4,5}$ para interferir com o processo físico-químico do desenvolvimento de lesões de cárie ${ }^{1}$. Há uma relação dose-efeito entre concentração de fluoreto em dentifrícios e redução de cárie ${ }^{3}$ e a concentração de no mínimo 1.000 ppm F de fluoreto solúvel continua sendo um parâmetro válido ${ }^{6,7}$, sendo que a FDI recomenda que um dentifrício mantenha após dois anos de fabricação pelo menos 800 ppm F de fluoreto solúvel ${ }^{8}$. Entretanto, a solubilidade do fluoreto em uma formulação de dentifrício depende do tipo de abrasivo e do sal de fluoreto usados.

Os dentifrícios mais consumidos, tanto no Brasi1 ${ }^{9,10}$ como nos países em desenvolvimento $^{11,12}$, são formulados com carbonato de cálcio $\left(\mathrm{CaCO}_{3}\right)$ como abrasivo e monofluorfosfato de sódio $\left(\mathrm{Na}_{2} \mathrm{FPO}_{3}\right.$; MFP) como componente ativo anticárie ${ }^{13}$. Esse tipo de formulação é relativamente estável no dentifrício fresco (recém-fabricado), mas em função do tempo de armazenamento, o íon MFP sofre hidrólise e o íon fluoreto liberado é insolubilizado pelo abrasivo, se tornando quimicamente inativo contra cárie. Por outro lado, devido ao menor custo de produção em relação a outras formulações que têm sílica como abrasivo, formulações à base de $\mathrm{CaCO}_{3} / \mathrm{MFP}$ têm impacto social para países em desenvolvimento devido ao seu baixo preço de mercado. Assim, essas formulações não são apenas usadas pelas famílias de menor nível sócio-econômico ${ }^{14}$, mas também prevalecemnas compras por licitações do Sistema Único de Saúde (SUS) e são distribuídas à população pelos serviços públicos de saúde do Brasil ${ }^{15}$. Assim, é reponsabilidade do governo garantir que a população tenha acesso a um dentifrício fluoretado com qualidade mínima em termos de benefício anticárie.

A legislação brasileira sobre a qualidade do fluoreto presente nos dentifrícios do mercado sofreu mudanças desde $1989^{15}$, culminando com a atual Resolução 79 da ANVISA de 28/08/2000 ${ }^{16}$. Esta apenas estabelece que um dentifrício não deve conter mais que $0,15 \%$ de fluoreto total $(1.500 \mathrm{ppm}$ $\mathrm{F} ; \mathrm{mg} \mathrm{F} / \mathrm{kg}$ ), sem especificar quanto desse fluoreto deve estar quimicamente solúvel na formulação fresca ou durante o prazo de validade para ter efeito anticárie. Como consequência, são encontrados à venda no mercado brasileiro, dentifrícios com concentração de fluoreto potencialmente ativo contra cárie muito abaixo do mínimo recomendado ${ }^{9}$. A situação se torna mais alarmante com relação aos dentifrícios distribuídos pelos serviços públicos de saúde do Brasil, do norte ao sul do país. Na maioria dos dentifrícios, são encontradas não só baixa concentração de fluoreto solúvel ativo contra cárie como até violação flagrante da ineficaz Resolução 79 da ANVISA com relação ao que é declarado pelo fabricante na rotulagem do produto e o que é de fato encontrado dentro dele.

Diante disso, este estudo tem como objetivo discutir que mudanças poderiam ser implementadas para que a população brasileira como um todo seja beneficiada pelo uso de dentifrícios fluoretados

ISSN 1982-8829 Tempus, actas de saúde colet, Brasília, 14(1), 09-27, mar, 2020. Epub Mai/2020 
em termos do controle de cárie dentária.

\section{MÉTODOS}

Uma busca na literatura foi realizada nas bases de dados Lilacs, PubMed e SciELO, tendo como intervalo de tempo o período de 1980 a 2019 e os seguintes descritores: dentifrícios, cremes dentais, fluoretos e Brasil, nos idiomas português e inglês. Os estudos foram inicialmente selecionados com base na leitura do título e resumo, utilizando critérios definidos, e lidos na íntegra para coleta de informações. Além das bases de dados já mencionadas, também foram consultados documentos oficiais do Ministério da Saúde e planilhas de dados do Laboratório de Bioquímica Oral da FOPUNICAMP.

Foram incluídos nessa revisão apenas os resultados de análises de dentifrícios contendo cálcio $\left(\mathrm{Ca}^{2+}\right)$ no abrasivo, que no Brasil são formulados com $\mathrm{CaCO}_{3}$ como abrasivo e MFP como sal de fluoreto, tendo em vista que as formulações fluoretadas contendo sílica como abrasivo são quimicamente estáveis ${ }^{13,17}$. Os resultados de todas as análises mostradas nas tabelas a seguir foram feitas em dentifrícios que estavam dentro do prazo de validade do produto declarada na embalagem, isto é, 3 ou menos anos de fabricação.

\section{ANÁLISE DE RESULTADOS}

\section{Qualidade do fluoreto nos dentifrícios encontrados no mercado}

Desde o início da década de 1980, os dentifrícios brasileiros têm sido analisados quanto à sua composição e concentração de fluoreto total (FT) e fluoreto solúvel total (FST; "ativo") ${ }^{18}$ para avaliar se os produtos à venda estão de acordo com as regulamentações brasileiras e ao mesmo tempo apresentam potencial para serem eficazes contra cárie. Na tabela 1, estão presentes dados de análises de concentração de FT e FST de dentifrícios à base de $\mathrm{CaCO}_{3} / \mathrm{MFP}$ encontrados no mercado brasileiro desde 1981 até o presente.

Em 1981, existiam no mercado brasileiro sete dentifrícios fluoretados, sendo dois formulados com MFP e abrasivos contendo $\mathrm{Ca}^{2+18}$. Ambos apresentavam menos de $900 \mathrm{ppm} \mathrm{F}$ na forma de FST, sendo que após um ano de armazenamento à temperatura ambiente foi encontrada uma concentração de FST três vezes menor ${ }^{19}$. Como mostra a tabela 1a (linha 3, coluna 5), quase a metade (45\%) do FT do dentifrício Kolynos SMF estava insolúvel, portanto “inativo” para ter efeito anticárie.

Em 1989, houve aumento da oferta de dentifrícios fluoretados no mercado brasileiro e 40\% deles eram formulados com abrasivo à base de $\mathrm{Ca}^{2+}$ e fluoreto como MFP ${ }^{20}$. Como mostra a tabela 1a (linhas 5-7), estes dentifrícios apresentavam baixas concentrações de FST baixas para terem efeito anticárie, sendo que em um deles mais de 50\% do FT estava quimicamente insolúvel, aumentando a porcentagem de $\mathrm{F}$ insolúvel após o envelhecimento (tabela 1a, última coluna, linhas 8-10).

Tempus, actas de saúde colet, Brasília, 13(3), 09-23, set, 2019. Epub Mai/2020 ISSN 1982-8829 
As análises descritas anteriormente foram feitas em dentifrícios comprados em Piracicaba, SP, região sudeste do Brasil, onde a maioria dos dentifrícios eram fabricados e havia dúvida sobre a qualidade do fluoreto de um mesmo dentifrício vendido nas 5 regiões do Brasil, a qual foi respondida em publicação feita em 1999²1. Nos dentifrícios recém-adquiridos (amostras frescas, tabela 1a, linhas 11-14), foi encontrada concentração de FST acima de 1.000 ppm F, independente da região do país onde eles foram adquiridos. Nas amostras frescas, já havia fluoreto insolúvel (9-27\%) e, após envelhecimento simulando 1 ano à temperatura ambiente ${ }^{22}$, a concentração de FST reduziu consideravelmente com o concomitante aumento da porcentagem de fluoreto insolúvel (35-52\%), quimicamente inativo para ter potencial anticárie (tabela 1a, linhas 15-18).

Em 2001, logo após a publicação da Resolução ${ }^{\circ} 79$ da ANVISA $^{16}$, foi publicado novo artigo sobre concentração de fluoreto nos cinco dentifrícios mais comercializados no Brasil e no qual foi incluído um dentifrício recém-lançado no comércio ${ }^{23}$. Todos os dentifrícios avaliados (tabela 1a, linhas 19-23) estavam de acordo com a Resolução ANVISA 79 quanto à concentração máxima de FT, entretanto na amostra fresca de todos já havia fluoreto inativo contra cárie, sendo que no produto recém-lançado (Contente) mais de 50\% do seu FT estava insolúvel.

Em 2003, foram publicados resultados da concentração e da estabilidade do fluoreto de 4 dentifrícios produzidos no Brasil e comercializados em Manaus (AM) ${ }^{17}$. Nas amostras recémadquiridas de todos os dentifrícios, havia concentração de FST com potencial anticárie (tabela 1a, linhas 24-27), mas já havia de 12 a 26\% de fluoreto insolúvel. Após um ano de armazenamento à temperatura ambiente (linhas 28-31), somente um dentifrício manteve concentração de FST acima de 1.000 ppm F.

Em 2010, Cury et al. (2010)9 avaliaram a concentração de fluoreto em dentifrícios, que estavam sendo utilizados por 207 crianças do município de Montes Claros, MG. Das 30 marcas de dentifrícios utilizados, 47\% estavam formulados com $\mathrm{CaCO}_{3} / \mathrm{MFP}$ e $46 \%$ destes continham menos de 1.000 ppm F na forma "ativa" de FST, sendo que em 2 deles mais de 50\% do FT estava insolúvel (tabela 1b, linhas 1-13).

A concentração de fluoreto nos 5 dentifrícios mais vendidos no Brasil voltou a ser analisada no início deste século ${ }^{10}$. Foram comprados dentifrícios nas 5 regiões do Brasil e 4 deles estavam formulados com $\mathrm{CaCO}_{3} / \mathrm{MFP}$. Nas amostras recém-adquiridas de todos os dentifrícios, concentração de FST superior a 1.000 ppm F (tabela 1b, linhas 14-17) foi encontrada, independente da região brasileira. Estes dentifrícios foram armazenados à temperatura ambiente e reavaliados ao final do prazo de validade de $3 \operatorname{anos}^{24}$, sendo constatada uma redução média de $28 \%$ na concentração de FST e consequente aumento de fluoreto insolúvel (tabela 1b, linhas 18-21). Essa publicação mostrou pela primeira vez que seria factível exigir dos fabricantes que seus dentifrícios mantivessem pelo prazo de fabricação a concentração de no mínimo 800 ppm de fluoreto potencialmente ativo contra cárie, isto é, quimicamente solúvel.

ISSN 1982-8829 Tempus, actas de saúde colet, Brasília, 13(3), 09-23, set, 2019. Epub Epub 
Os dentifrícios mais vendidos no Brasil são fabricados por multinacionais, mas também há inúmeros pequenos produtores de cremes dentais no país. Em publicação mais recente, foi feita uma análise destes dentifrícios produzidos por pequenos produtores e vendidos nas 5 regiões do país ${ }^{25}$. Foram analisados 12 dentifrícios à base de $\mathrm{CaCO}_{3} / \mathrm{MFP}$, sendo três da região norte, um da região nordeste, um da região centro-oeste, três da região sudeste, três da região sul e como "controle" foi comprado o dentifrício Sorriso Dentes Brancos em cada região. Um destes dentifrícios continha concentração de FT 9\% acima do limite máximo de 1.500 ppm F da Resolução n ${ }^{\circ} 79$ da ANVISA ${ }^{16}$. Em 5 deles, a concentração de FST encontrada nas amostras recém adquiridas era inferior a 1.000 ppm F, sendo que em um deles $60 \%$ do FT já estava insolúvel, portanto inativo contra cárie (tabela 1c, linhas 1-12).

Em 2019, foi comprado no comércio de Piracicaba, SP, o dentifrício marca FreeDent, o qual segundo a embalagem deveria conter 1.500 ppm F de FT, mas havia no mesmo apenas 555 ppm de FT (média de 2 bisnagas), do qual 13\% já estava insolúvel (tabela 1c, linha 13).

Tabela 1a. Concentração (ppm F; mg F/kg) de fluoreto total (FT) e de fluoreto solúvel total (FST = "potencialmente ativo contra cárie") encontrada nas análises e percentual de fluoreto insolúvel (\% F-ins = "inativo contra cárie") em dentifrícios comprados no Brasil. Brasil. 1981-2019. (continua).

\begin{tabular}{|c|c|c|c|c|}
\hline \multirow{2}{*}{ Ano } & \multirow{2}{*}{ Dentifrício } & \multicolumn{2}{|c|}{$\mathrm{mg} \mathrm{F} / \mathrm{kg}(\mathrm{ppm} \mathrm{F})$} & \multirow{2}{*}{$* \%$ F-ins. } \\
\hline & & FT & FST & \\
\hline \multirow{2}{*}{$\begin{array}{c}1981^{18} \\
\text { (Amostras frescas) }\end{array}$} & Kolynos SMF & 1010,8 & 819,6 & 18,9 \\
\hline & Colgate Fluorgard & 931,0 & 864,3 & 7,2 \\
\hline $1986^{19}$ & Kolynos SMF & 1124,2 & 614,7 & 45,3 \\
\hline $\begin{array}{l}\text { (Amostras } \\
\text { envelhecidas) }\end{array}$ & Colgate Fluorgard & 933,1 & 683,7 & 26,7 \\
\hline \multirow{3}{*}{$\begin{array}{c}1989^{20} \\
\text { (Amostras frescas) }\end{array}$} & Colgate Antiplaca & 394,3 & 359,3 & 8,9 \\
\hline & Colgate MFP & 499,9 & 230,6 & 59,3 \\
\hline & Flúor 2 & 915,6 & 815,6 & 10,9 \\
\hline \multirow{3}{*}{$\begin{array}{c}1989^{20} \\
\text { (Amostras } \\
\text { envelhecidas) }\end{array}$} & Colgate Antiplaca & 411,7 & 343,7 & 16,5 \\
\hline & Colgate MFP & 497,3 & 192,6 & 61,3 \\
\hline & Flúor 2 & 889,3 & 669,0 & 24,8 \\
\hline \multirow{4}{*}{$\begin{array}{c}1999^{21} \\
\text { (Amostras frescas) }\end{array}$} & Colgate Menta MFP $+\mathrm{Ca}$ & $1450 * *$ & 1061,7 & 26,8 \\
\hline & Signal Original & $1500 * *$ & 1365,4 & 9,0 \\
\hline & Gessy Lever & $1500 * *$ & 1259,4 & 16,0 \\
\hline & Kolynos Super Branco (KSB) & $1200 * *$ & 995,3 & 17,1 \\
\hline \multirow{4}{*}{$\begin{array}{c}1999^{21} \\
\text { (Amostras } \\
\text { envelhecidas) }\end{array}$} & Colgate Menta MFP $+\mathrm{Ca}$ & $1450 * *$ & 700,7 & 51,7 \\
\hline & Signal Original & $1500 * *$ & 766,9 & 48,9 \\
\hline & Gessy Lever & $1500 * *$ & 782,0 & 47,9 \\
\hline & Kolynos Super Branco (KSB) & $1200 * *$ & 781,2 & 34,9 \\
\hline
\end{tabular}




\begin{tabular}{|c|c|c|c|c|}
\hline \multirow{5}{*}{$\begin{array}{c}2001^{23} \\
\text { (Amostras frescas) }\end{array}$} & Colgate & 1321,6 & 1020,6 & 22,8 \\
\hline & Gessy Lever & 1443,3 & 1217,3 & 15,7 \\
\hline & Signal & 1394,0 & 1305,6 & 6,3 \\
\hline & Sorriso (antes chamado de KSB) & 1356,3 & 1164,9 & 14,1 \\
\hline & Contente & 1423,1 & 635,0 & 55,4 \\
\hline \multirow{4}{*}{$\begin{array}{c}2003^{17} \\
\text { (Amostras frescas) }\end{array}$} & Colgate MFP $+\mathrm{Ca}$ & 1508,5 & 1122,8 & 25,6 \\
\hline & Gessy & 1546,9 & 1363,6 & 11,8 \\
\hline & Signal & 1567,9 & 1375,7 & 12,2 \\
\hline & Sorriso & 1518,6 & 1243,2 & 18,1 \\
\hline \multirow{4}{*}{$\begin{array}{c}2003^{17} \\
\text { (Amostras } \\
\text { envelhecidas) }\end{array}$} & Colgate MFP $+\mathrm{Ca}$ & 1442,6 & 757,9 & 47,5 \\
\hline & Gessy & 1521,2 & 979,2 & 35,6 \\
\hline & Signal & 1544,2 & 1057,8 & 31,5 \\
\hline & Sorriso & 1507,2 & 795,6 & 47,2 \\
\hline
\end{tabular}

*[(FT-FST)/FT] x 100.

**Descrita na embalagem.

Tabela 1b. Concentração (ppm F; mg F/kg) de fluoreto total (FT) e de fluoreto solúvel total $(\mathrm{FST}=$ "potencialmente ativo contra cárie") encontrada nas análises e percentual de fluoreto insolúvel (\% F-ins = "inativo contra cárie") em dentifrícios comprados no Brasil. Brasil. 19812019. (continuação).

\begin{tabular}{|c|c|c|c|c|}
\hline \multirow{2}{*}{ Ano } & \multirow{2}{*}{ Dentifrício } & \multicolumn{2}{|c|}{$\mathrm{mg} \mathrm{F} / \mathrm{kg}(\mathrm{ppm} \mathrm{F})$} & \multirow{2}{*}{$\%$ F-ins. } \\
\hline & & FT & FST & \\
\hline \multirow{13}{*}{$\begin{array}{c}2010^{9} \\
\text { (Amostras } \\
\text { frescas) }\end{array}$} & Sorriso Dentes Brancos & 1435,3 & 1122,8 & 21,8 \\
\hline & Colgate Máxima Proteção Anticárie & 1438,6 & 862,6 & 40,0 \\
\hline & Colgate Tripla Ação & 1470,9 & 1082,6 & 26,4 \\
\hline & Onda Fresh & 1787,1 & 1013,1 & 43,3 \\
\hline & Close-Up Triple & 1443,3 & 1228,6 & 14,9 \\
\hline & Contente Kids & 1105,0 & 529,0 & 52,1 \\
\hline & Dentil Kids & 905,3 & 422,3 & 53,4 \\
\hline & Contente Plus & 1606,4 & 1023,5 & 36,3 \\
\hline & IceFresh & 1602,1 & 887,2 & 44,6 \\
\hline & Sorriso Herbal & 1490,9 & 1049,7 & 29,6 \\
\hline & Colgate Herbal & 1455,2 & 1074,9 & 26,1 \\
\hline & Condor $100 \%$ Branco & 1550,6 & 914,8 & 41,0 \\
\hline & Colgate Cavity Protection & 955,2 & 748,6 & 21,6 \\
\hline \multirow{4}{*}{$\begin{array}{c}2012^{10^{*}} \\
\text { (Amostras } \\
\text { frescas) }\end{array}$} & Colgate Máxima Proteção Anticáries & 1433,6 & 1135,3 & 20,8 \\
\hline & Colgate Tripla Ação Menta Original & 1433,2 & 1160,9 & 19,0 \\
\hline & Colgate Tripla Ação Menta Suave & 1430,1 & 1122,3 & 21,5 \\
\hline & Sorriso Dentes Brancos & 1441,2 & 1098,1 & 23,8 \\
\hline \multirow{4}{*}{$\begin{array}{c}2015^{24^{*}} \\
\text { (Ao final } \\
\text { do prazo de } \\
\text { validade de } 3 \\
\text { anos) }\end{array}$} & Colgate Máxima Proteção Anticáries & 1411,2 & 756,5 & 46,4 \\
\hline & Colgate Tripla Ação Menta Original & 1411,9 & 858,5 & 39,2 \\
\hline & Colgate Tripla Ação Menta Suave & 1396,4 & 799,7 & 42,7 \\
\hline & Sorriso Dentes Brancos & 1442,1 & 831,6 & 42,3 \\
\hline
\end{tabular}

*Dentifrícios mais vendidos no Brasil e comprados nas 5 regiões do país.

ISSN 1982-8829 Tempus, actas de saúde colet, Brasília, 14(1), 09-27, mar, 2020. Epub Mai/2020 
Tabela 1c. Concentração (ppm F; mg F/kg) de fluoreto total (FT) e de fluoreto solúvel total (FST $=$ "potencialmente ativo contra cárie") encontrada nas análises e percentual de fluoreto insolúvel (\% F-ins = "inativo contra cárie”) em dentifrícios comprados no Brasil. Brasil. 19812019. (conclusão).

\begin{tabular}{|c|c|c|c|c|}
\hline \multirow{2}{*}{ Ano } & \multirow{2}{*}{ Dentifrício } & \multicolumn{2}{|c|}{$\mathrm{mg} \mathrm{F} / \mathrm{kg}(\mathrm{ppm} \mathrm{F})$} & \multirow{2}{*}{$\%$ F-ins } \\
\hline & & FT & FST & \\
\hline \multirow{12}{*}{ (Amostras frescas) } & Even Dentes Fortes (N) & 1513,8 & 1120,4 & 26,0 \\
\hline & Even 2 em $1(\mathrm{~N})$ & 1530,9 & 1198,5 & 21,7 \\
\hline & Ultra Action (N) & 1295,1 & 502,3 & 61,2 \\
\hline & Even Dentes Fortes (NE) & 1435,3 & 797,5 & 44,4 \\
\hline & Contente Plus (CO) & 1534,2 & 755,9 & 50,7 \\
\hline & Dentil (SE) & 1207,9 & 701,0 & 42,0 \\
\hline & FreeDent (SE) & 1348,1 & 1309,6 & 2,9 \\
\hline & Ultra Action (SE) & 1218,2 & 689,2 & 43,4 \\
\hline & Anticáries Dia (S) & 1424,0 & 1412,4 & 0,8 \\
\hline & IceFresh (S) & 1638,2 & 1161,0 & 29,1 \\
\hline & Ultra Action (S) & 1286,2 & 1060,2 & 17,6 \\
\hline & Sorriso & 1502,2 & 1227,2 & 18,3 \\
\hline $\begin{array}{c}2019 \\
\text { (Amostra fresca) }\end{array}$ & FreeDent & 555,2 & 483,1 & 13,0 \\
\hline
\end{tabular}

$\S$ Dentifrícios fluoretados comprados nas 5 regiões do Brasil: Norte (N), Nordeste (NE), Centro-Oeste (CO), Sudeste (SE) e Sul (S), comparados com o dentifrício mais consumido no Brasil (Sorriso).

\section{Qualidade do fluoreto nos dentifrícios distribuídos pelo SUS}

Os dentifrícios adquiridos pelo SUS são comprados por licitações e distribuídos à população pelas Unidades Básicas. Como mencionado na introdução desse artigo, geralmente são formulados com $\mathrm{CaCO}_{3} / \mathrm{MFP}$ devido ao baixo custo desta formulação quando comparados a dentifrícios que contêm sílica como abrasivo. Como também já mencionado, esse tipo de formulação é quimicamente compatível quando da fabricação do dentifrício, mas não é estável durante todo o tempo de validade do dentifrício porque a concentração de fluoreto solúvel diminui com o concomitante aumento de fluoreto insolúvel, o qual não tem potencial anticárie. Por essa razão, os dentifrícios com $\mathrm{CaCO}_{3} /$ MFP são geralmente formulados com a concentração máxima de fluoreto total permitida pela Resolução 79 da ANVISA, que é de 1.500 ppm F.

O Laboratório de Bioquímica Oral da FOP-UNICAMP realiza há mais de 30 anos um trabalho de extensão universitária, fazendo gratuitamente análise química de dentifrícios que são distribuídos pelos serviços públicos de saúde do Brasil.Os solicitantes são colegas cirurgiões-dentistas (CD) trabalhadores do SUS ou coordenadores de saúde bucal, para os quais são enviados laudos os alertando para a qualidade dos dentifrícios em uso. A tabela 2 é um resumo dos laudos que foram encontrados nos arquivos do laboratório ou de relatórios, caso da análise feita nos dentifrícios que estavam sendo distribuídos pelo programa Brasil Sorridente em 2010. 
À primeira vista, o que chama a atenção nesses dentifrícios distribuídos pelo SUS (tabela 2) não é o fato que eles também apresentam fluoreto inativo contra cárie (\% F-ins, última coluna), mas sim que quase $50 \%$ dos dentifrícios analisados sequer estavam de acordo com a Resolução ${ }^{\circ} 79$ da ANVISA $^{16}$, pois continham mais de 1.500 ppm F de FT. Além dessa violação por excesso de FT e pouco fluoreto ativo contra cárie (FST), outro fato que chama a atenção é a fraude no processo de licitação. Assim, no dentifrício distribuído pelo SUS em Salvador, BA, embora na embalagem do produto constasse que ele foi fluoretado (1.500 ppm F como MFP), nada de MFP foi encontrado no mesmo (tabela $2 \mathrm{~b}$, linha 16).

Até mesmo a Coordenação de Saúde Bucal do Ministério da Saúde foi atingida pela ineficácia do processo de licitação de compra dos dentifrícios que eram distribuídos pelo Brasil.No ano de 2011, o Laboratório de Bioquímica Oral da FOP-UNICAMP firmou acordo com a referida Coordenação para analisar a qualidade do fluoreto dos dentifrícios que estavam sendo distribuídos pelo programa Brasil Sorridente em todas as regiões dopaís. Nas amostras frescas recebidas, chamou a atenção que em termos de FT havia diferença entre os dentifrícios distribuídos. Nas regiões Norte, Nordeste e Sul foram distribuídos dentifrícios formulados com maior concentração de FT que os das regiões Centro-Oeste e Sudeste (tabela 2a, coluna 4, linhas 15-19). Com relação à concentração de fluoreto potencialmente ativo contra cárie (FST), apenas os dentifrícios que estavam sendo distribuídos nas regiões Norte e Sul tinham mais de 1.000 ppm F. Após 1 ano de armazenamento em Piracicaba, $\mathrm{SP}$, a porcentagem de fluoreto inativo contra cárie (\%F-ins) aumentou em todos (tabela $2 \mathrm{~b}$, última coluna, linhas 1-5).

Os dados da tabela 2 mostram que a qualidade dos dentifrícios fluoretados distribuídos pelo SUS no Brasil independe da região ou estado do país, pois o mesmo problema é constatado do RS (Santiago) ao AM (Manaus, São Gabriel da Cachoeira), passando por SP (Igarapava, Salto de Pirapora, São João da Boa Vista, Santos). Entretanto, o fato mais grave do ponto de vista de saúde pública foi o constatado em São Gabriel da Cachoeira, AM, pois nenhum dos dois dentifrícios que estavam sendo distribuídos para a população indígena local tinha potencial anticárie, quer seja o chancelado pelo Ministério da Saúde (Brasil Sorridente) como o dentifrício comercial Contente (Tabela 2b, coluna 5, linhas 6-7). Na realidade, esses dentifrícios nem mesmo atendiam a desatualizada Portaria 79 da ANVISA, pois embora deficientes de fluoreto ativo contra cárie, tinham mais de 1.500 ppm F de FT (Tabela 2b, coluna 4).

Tabela 2a. Concentração (ppm F; mg F/kg) de fluoreto total (FT) e de fluoreto solúvel total (FST = "potencialmente ativo contra cárie") encontrada nas análises e percentual de fluoreto insolúvel (\%F-ins = "inativo contra cárie") em dentifrícios distribuídos à população por Serviços Públicos de Saúde do Brasil. Brasil. 2000-2019. (continua).

\begin{tabular}{|l|l|l|l|l|l|}
\hline \multirow{2}{*}{ Ano } & \multirow{2}{*}{ Origem } & \multirow{2}{*}{ Dentifrício } & \multicolumn{2}{|c|}{$\mathrm{mg} \mathrm{F} / \mathrm{kg}(\mathrm{ppm} \mathrm{F})$} & \multirow{2}{*}{$\%$ F-ins. } \\
\cline { 3 - 4 } & & FT & FST & \\
\hline
\end{tabular}

ISSN 1982-8829 Tempus, actas de saúde colet, Brasília, 14(1), 09-27, mar, 2020. Epub Mai/2020 


\begin{tabular}{|c|c|c|c|c|c|}
\hline \multirow{2}{*}{2000} & \multirow{2}{*}{$\begin{array}{l}\text { São João da Boa } \\
\text { Vista, SP }\end{array}$} & Contente & 1462,6 & 553,4 & 62,2 \\
\hline & & Contente & 1463,1 & 363,1 & 75,2 \\
\hline \multirow{4}{*}{2001} & \multirow{4}{*}{ Serra, ES } & Action & 1211,5 & 953,6 & 21,3 \\
\hline & & Nevasca & 1318,0 & 1087,7 & 17,5 \\
\hline & & Contente Plus & 1639,1 & 1150,8 & 29,8 \\
\hline & & Colgate & 1390,3 & 1235,2 & 11,2 \\
\hline \multirow{2}{*}{2004} & \multirow{2}{*}{ Uberlândia, MG } & Ki-pasta & 1588,4 & 819,3 & 48,4 \\
\hline & & Contente & 1725,2 & 1055,8 & 38,8 \\
\hline \multirow{2}{*}{2007} & \multirow{2}{*}{ Brasília, DF } & IceFresh & 1523,5 & 1082,8 & 28,9 \\
\hline & & Creme Dental do Kit PSF & 1520,3 & 1058,2 & 30,4 \\
\hline \multirow{3}{*}{2008} & \multirow{2}{*}{ Brasília, DF } & Action & 1242,2 & 1204,6 & 3,0 \\
\hline & & Action (amostraenvelhecida) & 1266,4 & 925,4 & 26,9 \\
\hline & Santos, SP & IceFresh & 1576,1 & 852,5 & 45,9 \\
\hline 2009 & Teresina, PI & Brasil Sorridente & 1503,2 & 989,4 & 34,2 \\
\hline \multirow{5}{*}{2011} & \multirow{5}{*}{$\begin{array}{l}\text { Brasil } \\
\text { (Amostras frescas) }\end{array}$} & $\begin{array}{l}\text { Brasil Sorridente } \\
\text { (Belém, PA) }\end{array}$ & 1595,2 & 1109,8 & 30,4 \\
\hline & & $\begin{array}{l}\text { Brasil Sorridente } \\
\text { (Junqueira,AL) }\end{array}$ & 1564,8 & 833,5 & 46,7 \\
\hline & & $\begin{array}{l}\text { Brasil Sorridente } \\
\text { (Goiânia, GO) }\end{array}$ & 1220,5 & 661,1 & 45,8 \\
\hline & & $\begin{array}{l}\text { Brasil Sorridente } \\
(\text { Embu, SP) }\end{array}$ & 1229,4 & 683,9 & 44,4 \\
\hline & & $\begin{array}{l}\text { Brasil Sorridente } \\
\text { (Maringá, PR) }\end{array}$ & 1648,8 & 1063,4 & 35,5 \\
\hline
\end{tabular}

Fonte: Arquivos do Laboratório de Bioquímica Oral, FOP-UNICAMP. 
Tabela 2b. Concentração (ppm F; mg F/kg) de fluoreto total (FT) e de fluoreto solúvel total (FST = "potencialmente ativo contra cárie") encontrada nas análises e percentual de fluoreto insolúvel (\%F-ins = "inativo contra cárie") em dentifrícios distribuídos à população por Serviços Públicos de Saúde do Brasil. Brasil. 2000-2019. (conclusão).

\begin{tabular}{|c|c|c|c|c|c|}
\hline \multirow{2}{*}{ Ano } & \multirow{2}{*}{ Origem } & \multirow{2}{*}{ Dentifrício } & \multicolumn{2}{|c|}{ mg F/kg (ppm F) } & \multirow{2}{*}{$\%$ F-ins. } \\
\hline & & & FT & FST & \\
\hline \multirow{5}{*}{2011} & \multirow{5}{*}{$\begin{array}{l}\text { Brasil } \\
\text { (Amostras envelhecidas) }\end{array}$} & $\begin{array}{l}\text { Brasil Sorridente } \\
\text { (Belém, PA) }\end{array}$ & 1597,4 & 1087,3 & 31,9 \\
\hline & & $\begin{array}{l}\text { Brasil Sorridente } \\
\text { (Junqueira, AL) }\end{array}$ & 1611,4 & 510,9 & 68,3 \\
\hline & & $\begin{array}{l}\text { Brasil Sorridente } \\
\text { (Goiânia, GO) }\end{array}$ & 1306,9 & 492,0 & 62,4 \\
\hline & & $\begin{array}{l}\text { Brasil Sorridente } \\
\text { (Embu, SP) }\end{array}$ & 1265,0 & 536,0 & 57,6 \\
\hline & & $\begin{array}{l}\text { Brasil Sorridente } \\
\text { (Maringá, PR) }\end{array}$ & 1675,5 & 943,1 & 43,7 \\
\hline \multirow{2}{*}{2011} & \multirow{2}{*}{$\begin{array}{l}\text { São Gabriel da Cachoeira, } \\
\text { AM }\end{array}$} & Brasil Sorridente & 1585,3 & 243,9 & 84,6 \\
\hline & & Contente & 1663,0 & 694,7 & 58,2 \\
\hline 2015 & Balneário Camboriú, SC & IceFresh & 1640,5 & 546,5 & 66,7 \\
\hline \multirow{3}{*}{2017} & \multirow{2}{*}{ Rio Claro, SP } & Escovinha Kids & 1171,2 & 731,1 & 37,6 \\
\hline & & Trá Lá Lá Kids & 1189,0 & 743,4 & 37,5 \\
\hline & Salto de Pirapora, SP & Contente & 1552,7 & 802,7 & 48,3 \\
\hline \multirow{2}{*}{2018} & \multirow{2}{*}{ Manaus, AM } & Contente & 1528,6 & 783,4 & 48,8 \\
\hline & & IceFresh & 1708,3 & 824,9 & 51,7 \\
\hline \multirow{5}{*}{2019} & Piracicaba, SP & $\begin{array}{l}\text { AME Menta } \\
\text { Refrescante }\end{array}$ & 1408,8 & 654,7 & 53,5 \\
\hline & Igarapava, SP & FreeDent & 1034,7 & 876,5 & 15,3 \\
\hline & Salvador, BA & FreeDent & $0,0^{*}$ & 0,0 & 0,0 \\
\hline & \multirow{2}{*}{ Santiago, RS } & FreeDent & 982,8 & 955,5 & 2,8 \\
\hline & & IceFresh & 1251,4 & 574,6 & 54,1 \\
\hline
\end{tabular}

Fonte: Arquivos do Laboratório de Bioquímica Oral, FOP-UNICAMP.

*Dentifrício não fluoretado (traços de fluoreto foram encontrados).

\section{Regulamentação brasileira sobre dentifrícios fluoretados: 1989-presente}

A vigilância em saúde pode ser considerada no Brasil como um dos mais importantes componentes na organização e gestão dos serviços do Sistema Único de Saúde (SUS) ${ }^{26,27}$. Nesse contexto, a Vigilância Sanitária apresenta-se como um sistema permanente mantido pelo Poder Público de ações articuladas, que orientam a redução e eliminação dos riscos à saúde decorrentes dos processos de produção, distribuição e consumo de bens e serviços ${ }^{26}$. No âmbito da saúde bucal, as ações da Vigilância Sanitária abrangem principalmente três eixos: a) alimentos, bebidas e medicamentos; b) estabelecimentos de prestação de serviços odontológicos; e c) produtos para higiene bucal, nos quais estão os dentifrícios fluoretados. 
Como relatado, as concentrações de fluoreto (total e solúvel) em dentifríciosno Brasil têm sido avaliadas desde o início da década de $1980^{18}$. Entretanto, apenas em 1989 foi estabelecida uma regulamentação brasileira para os dentifrícios fluoretados. A Portaria $n^{0} 22$ da Secretaria Nacional de Saúde de Vigilância Sanitária ${ }^{28}$ estabelecia parâmetros máximos e mínimos de concentração de fluoreto solúvel que um dentifrício deveria apresentar. Assim, quando fabricados (amostra fresca), os dentifrícios deveriam ter uma concentração de fluoreto solúvel de no mínimo 1.100 ppm F e no máximo de 1.500 ppm F. Também foram estabelecidas concentrações mínimas de fluoreto solúvel que um dentifrício deveria manter durante o prazo de validade. Essa portaria sofreu diversas modificações e foi substituída por regulamentações posteriores, como observado na linha do tempo da Figura 1.

Figura 1. Linha do tempo da regulamentação brasileira sobre dentifrícios fluoretados.

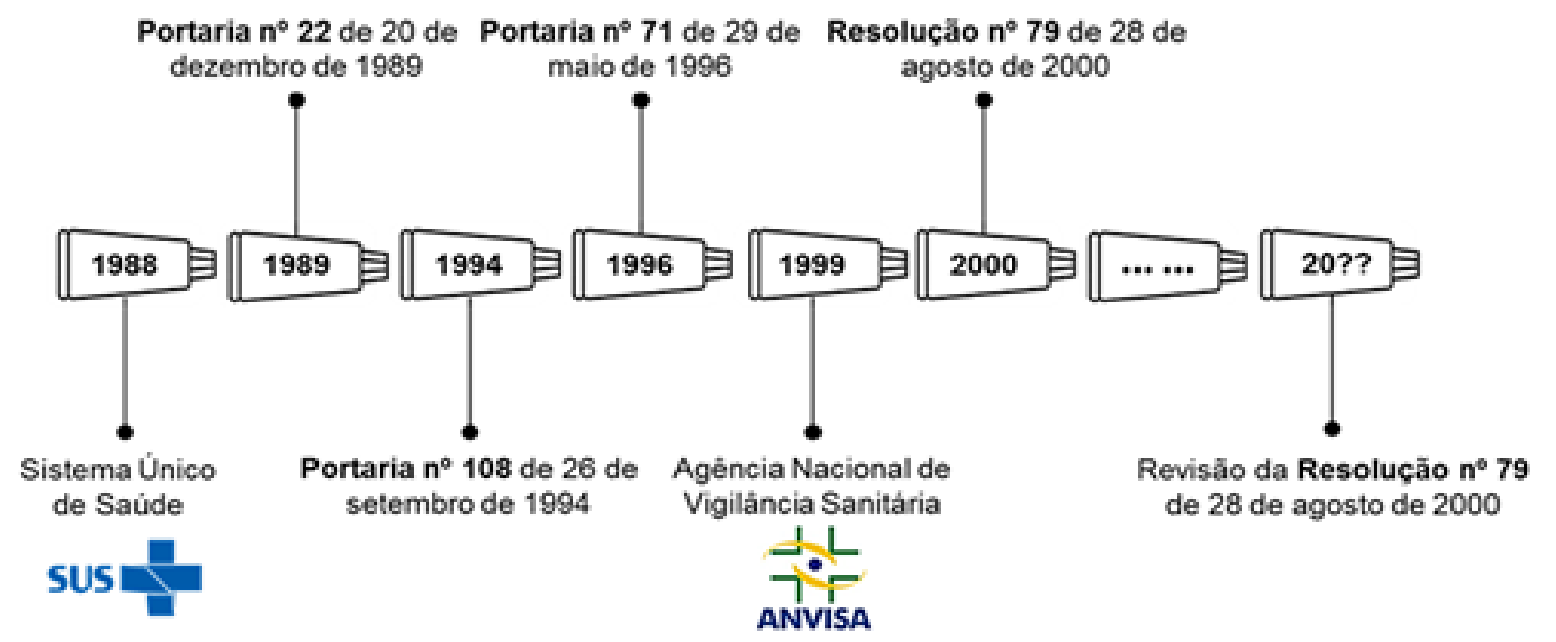

No ano de 1994, a Portaria $n^{0} 22$ foi revogada, entrando em vigor a Portaria $n^{\circ} 108$ de 26 de setembro de $1994^{29}$. Essa portaria manteve as mesmas especificações da anterior quanto à concentração e estabilidade do fluoreto que um dentifrício deveria conter quando fabricado e durante seu prazo de validade. Contudo, a portaria apenas se referia à concentração de fluoreto em ppm (mg F/kg) sem especificar a necessidade de estar solúvel. Com isso, passou a existir o risco de um dentifrício ser completamente ineficaz no controle da cárie dentária mesmo contendo fluoreto de acordo com as especificações da regulamentação, uma vez que se houvesse no mercado um dentifrício com no máximo $1.500 \mathrm{ppm}$ de fluoreto total, mas totalmente insolúvel, a portaria estaria sendo atendida.

Uma nova portaria foi regulamentada em 1996, a Portaria $n^{0} 71$ de 29 de maio de $1996^{30}$. Assim como as demais regulamentações, ela estabelecia que um dentifrício deveria conter uma concentração de no máximo 1.500 ppm F (0,15\% de fluoreto). Porém, não especificava a necessidade do fluoreto estar em uma forma quimicamente solúvel. Além disso, a Portaria $\mathrm{n}^{\mathrm{o}} 71$ descrevia uma variedade de sais de fluoreto que poderiam ser utilizados em dentifrícios, incluindo até mesmo os de baixíssima solubilidade, como o fluoreto de cálcio $\left(\mathrm{CaF}_{2}\right)$.

Tempus, actas de saúde colet, Brasília, 14(1), 09-27, mar, 2020. Epub Mai/2020 ISSN 1982-8829 
Com a criação da Agência Nacional de Vigilância Sanitária (ANVISA) em 1999, no ano seguinte entrou em vigor a Resolução $\mathrm{n}^{\circ}$ 79, de 28 de agosto de 2000 da ANVISA ${ }^{16}$. Essa resolução encontrase vigente até os dias atuais e determina que a concentração máxima total fluoreto presente nas formulações não deve exceder $0,15 \%$ expresso em fluoreto (1.500 ppm F), além de especificaros diversos sais de fluoreto que podem ser utilizados em dentifrícios. Entretanto, a Resolução no 79 da ANVISA não regulamenta o quanto de fluoreto solúvel um dentifrício deveria conter e manter pelo seu prazo de validade.

O histórico da regulamentação dos dentifrícios fluoretados no Brasil (Figura 1) mostra que apenas a questão relacionada à segurança do uso do fluoreto tem sido considerada. Dessa forma, há quase 20 anos a população brasileira encontra-se "refém" de uma resolução que apenas especifica o máximo de fluoreto que uma formulação deve conter (1.500 ppm F), sem nenhuma garantia de que esse fluoreto esteja solúvel por determinado período de tempo e tenha efeito no controle da cárie dentária. Diante disso, diversos estudos têm sido publicados na perspectiva de ratificar a necessidade de revisão da norma brasileira sobre dentifrícios fluoretados ${ }^{10,15,17,23-25,31,32}$.

O relatado nessa discussão não é uma especificidade do Brasil, mas sim um problema mundialsinalizado pela World Dental Federation (FDI) ${ }^{8}$. As regulamentações de diversos países do mundo não garantem que um dentifrício tenha uma concentração mínima de fluoreto solúvel para o controle da cárie dentária, como atestado em publicações internacionais ${ }^{11,33-38}$. As normas da União Europeia ${ }^{39}$ e do MERCOSUL ${ }^{40}$, à semelhança da Resolução $n^{\circ} 79$ da ANVISA, apenas

estabelecem o máximo de fluoreto total que um dentifrício deve conter $(0,15 \%)$. À exceção, as legislações do $\mathrm{EUA}^{41}$ e de Madagascar ${ }^{42}$, além de estabelecerem o valor máximo de fluoreto total que os dentifrícios devem conter, requerem que eles tenham uma concentração mínima de fluoreto solúvel por determinado período de tempo de fabricação do produto.

\section{DISCUSSÃO GERAL E PROPOSTA DE MUdANÇAS NA RESOLUÇÃO 79}

O presente trabalho detalha, atualiza e ratifica a publicação de Cury et al. (2015) $)^{15}$ sobre a necessidade de revisão da Resolução $n^{0} 79$ da ANVISA, porque não basta ela determinar que um dentifrício não pode conter mais que $0,15 \%$ (1.500 ppm) de fluoreto total. Essa concentração foi estabelecida com base na preocupação de segurança de produto de livre venda e risco de fluorose dental devido à ingestão de dentifrícios por crianças. Além dela não estar sendo cumprida, porque como mostramos (Tabelas 1 e 2) há no mercado produtos com até 1.700 ppm de FT, mas muito pouco fluoreto solúvel para ter efeito anticárie. Em acréscimo, cárie continua sendo um problema de saúde pública, afetando mais de 2,5 bilhões de pessoas ao redor do mundo e impactando mais a qualidade de vida das pessoas que fluorose dental, mesmo em países desenvolvidos como Austrália ${ }^{43}$ e EUA ${ }^{44}$ que à semelhança do Brasil convivem com água fluoretada e uso de dentifrício fluoretado.

Por outro lado, a razão maior para que a Portaria 79 sobre dentifrícios precisa ser mudada é o fato que os mais prejudicados por ela tem sido os que mais necessitam da qualidade do fluoreto 
para prevenção de cárie, que são as pessoas de menor poder aquisitivo e que dependem de políticas públicas de distribuição de dentifrícios. Esses são comprados pelos serviços públicos de saúde por meio de um processo de licitação pública e os fabricantes estão respaldados por uma Portaria que não protege o cidadão. À prova disso, o laboratório de Bioquímica Oral da FOP-UNICAMP analisou há 2 anos um dentifrício que venceu uma licitação e ia ser distribuído para crianças de escolas públicas de cidade de SP. Nosso laudo afirmava:

"Esse dentifrício apresenta uma concentração de flúor total não superior a 1.500 ppm F, que é o máximo permitido pela Resolução 79 da ANVISA, entretanto apresenta menos de 1.000 ppm $F$ de flúor solúvel que é de acordo com a melhor evidência científica disponível, o mínimo para ter beneficio anticárie"

Com base na Resolução 79, a firma que ganhou a licitação contestou judicialmente o laudo, afirmando:

"Neste sentido cumpre ressaltar que o Creme Dental X foi submetido à análise perante a Anvisa, sendo que o mesmo foi devidamente aprovado por aquela Agência, no dia DD/M/A, por meio da Resolução No. Y, conforme comprova o documento anexo.

Percebe-se, portanto, que a concentração e quantidade de flúor disponivel no referido Creme Dental corrobora as normas editadas pela própria Anvisa, pelo que não há que se falar em qualquer irregularidade no produto"

Assim, a presente Resolução ANVISAsobre dentifrícios precisa ser mudada no sentido que ela contemple os benefícios da escovação dos dentes com dentifrício adequadamente fluoretado, bastando exigir do fabricante:

1. Que o dentifrício não contenha mais que $1.500 \mathrm{ppm}(\mathrm{mg} / \mathrm{kg})$ de fluoreto total (FT)

2. Que pelo menos 1.000 ppm do FT esteja quimicamente solúvel (FST) na amostra fresca (recém-fabricada)

3. Que o dentifrício mantenha pelo menos $800 \mathrm{ppm}$ de fluoreto solúvel (FST) pelo prazo de 2 anos de fabricação do produto

A razão de máximo de 1.500 ppm de FT é por questão de segurança de produto de livre venda, a de 1.000 ppm de fluoreto solúvel é para garantir um efeito mínimo anticárie e 800 ppm solúvel por 2 anos é por ser uma concentração factível de ser obtida até pelos pequenos produtores brasileiros de creme dental, não os alijando da competição com as multinacionais, mesmo porque são eles que 
ganham as licitações feitas pelo SUS-Brasil.

Essa mudança visa garantir que todo brasileiro não só use um dentifrício fluoretado seguro, mas principalmente que nenhum cidadão brasileiro corra o risco de consumir um dentifrício fluoretado ineficaz no controle da cárie dentária, como demonstrado nesse trabalho.

\section{REFERÊNCIAS BIBLIOGRÁFICAS}

1. Cury JA, Tenuta LMA. Enamel remineralization: controlling the caries disease or treating early caries lesions? Braz Oral Res. 2009;23 Suppl 1:23-30.

2. Cury JA, Tenuta LMA. Evidence-based recommendation on toothpaste use. Braz Oral Res. 2014;28 Spec No:1-7. doi: 10.1590/S1806-83242014.50000001.

3. Walsh T, Worthington HV, Glenny AM, Marinho VCC, Jeroncic A. Fluoride toothpastes of different concentrations for preventing dental caries. Cochrane Database Syst Rev. 2019 Mar 4;3:CD007868. doi: 10.1002/14651858.CD007868.pub3.

4. Stookey GK. Are all fluoride dentifrices the same? In: Wei SHY, editor. Clinical uses of fluorides. Philadelphia: Lea \& Febiger; 1985:105-31.

5. Tenuta LMA, Cury JA. Laboratory and human studies to estimate anticaries efficacy of fluoride toothpastes. Monogr Oral Sci. 2013;23:108-24. doi: 10.1159/000350479.

6. Walsh T, Worthington HV, Glenny AM, Appelbe P, Marinho VCC, Shi X. Fluoride toothpastes of different concentrations for preventing dental caries in children and adolescents. Cochrane Database Syst Rev. 2010 Jan 20;(1):CD007868. doi: 10.1002/14651858.CD007868. pub2.

7. dos Santos AP, Nadanovsky P, de Oliveira BH. A systematic review and meta-analysis of the effects of fluoride toothpastes on the prevention of dental caries in the primary dentition of preschool children. Community Dent Oral Epidemiol. 2013 Feb;41(1):1-12. doi: 10.1111/j.16000528.2012.00708.x.

8. FDI. Promoting Dental Health through Fluoride Toothpaste. 2018 [citado 2019 set 30]. Disponível em: https://www.fdiworlddental.org/resources/policy-statements/promoting-dentalhealth-through-fluoride-toothpaste.

9. Cury JA, Oliveira MJL, Martins CC, Tenuta LMA, Paiva SM. Available fluoride in 
toothpastes used by Brazilian children. Braz Dent J. 2010;21(5):396-400.

10. Ricomini Filho AP, Tenuta LMA, Fernandes FSF, Calvo AFB, Kusano SC, Cury JA. Fluoride concentration in the top-selling Brazilian toothpastes purchased at different regions. Braz Dent J. 2012;23(1):45-8.

11. Giacaman RA, Carrera CA, Muñoz-Sandoval C, Fernandez C, Cury JA. Fluoride content in toothpastes commercialized for children in Chile and discussion on professional recommendations of use. Int J Paediatr Dent. 2013 Mar;23(2):77-83. doi: 10.1111/j.1365-263X.2012.01226.x.

12. Vorster L, Naidoo S, Stauf N, Holmgren C, Benzian H. Fluoride content of toothpastes available in South Africa. Community Dent Health. 2018 Aug 30;35(3):186-92. doi: 10.1922/ CDH_4294Vorster07.

13. Lippert F. An introduction to toothpaste - its purpose, history and ingredients. Monogr Oral Sci. 2013;23:1-14. doi: 10.1159/000350456.

14. Martins CC, Oliveira MJ, Pordeus IA, Cury JA, Paiva SM. Association between socioeconomic factors and the choice of dentifrice and fluoride intake by children. Int J Environ Res Public Health. 2011 Nov;8(11):4284-99. doi: 10.3390/ijerph8114284.

15. Cury JA, Caldarelli PG, Tenuta LMA. Necessity to review the Brazilian regulation about fluoride toothpastes. Rev Saude Publica. 2015;49. pii: S0034-89102015000100407. doi: 10.1590/ S0034-8910.2015049005768.

16. Brasil. Ministério da Saúde. Agência Nacional de Vigilância Sanitária (ANVISA). Resolução n 79 , de 28 de Agosto de 2000. Diário Oficial da União. 31 ago 2000. pp. 1415-1537.

17. Conde NCO, Rebelo MAB, Cury JA. Evaluation of the fluoride stability of dentifrices sold in Manaus, AM, Brazil. Pesqui Odontol Bras. 2003 Jul-Sep;17(3):247-53.

18. Cury JA, Guimarães LOC, Arbex ST, Moreira BW. Análise de dentifrícios fluoretados: concentração e formas químicas de fluoretos encontrados em produtos brasileiros. Rev Assoc Paul Cir Dent. 1981 Mar-Abr;35(2):142-7.

19. Cury JA. Estabilidade do flúor nos dentifrícios brasileiros. Rev Gaúcha Odontol. 1986 SetOut;34(5):430-2.

20. Cury JA. Dentifrícios fluoretados no Brasil. Rev Gaúcha Odontol. 1989 Mar- 
Abr;37(2):139-42.

21. Duarte FF, Pisaneschi E, Cury JA. Avaliação do flúor dos dentifrícios mais consumidos no Brasil e comercializados nas cinco regiões do país. Rev ABOPREV.1999;2(2):3-10.

22. Tabchoury CPM, Cury JA. Estudo de condições de envelhecimento precoce de dentifrícios para prever o comportamento de flúor em condições ambientais. Rev Bras Farm. 1994;75(3):67-71.

23. Orth RM, Assaf AV, Zanin L, Mialhe FL, Klein ALL, Medina MRJ, et al. Concentração de flúor nos principais dentifrícios comercializados no Brasil e impacto da nova portaria de regulamentação. Rev Odonto Cienc. 2001;16(32):27-33.

24. Cury JA, Dantas EDV, Tenuta LMA, Romão DA, Tabchoury CPM, Nóbrega DF et al. Concentração de fluoreto nos dentifrícios a base de $\mathrm{MFP} / \mathrm{CaCO}_{3}$ mais vendidos no Brasil, ao final dos seus prazos de validade. Rev Assoc Paul Cir Dent. 2015 Set;69(3):248-51.

25. Marín LM, Vieira W, Tenuta LMA, Tabchoury CPM, Cury JA. Concentração de fluoreto nos dentifrícios vendidos localmente no Brasil. Rev Assoc Paul Cir Dent. 2017;71(1):60-5.

26. Brasil. Ministério da Saúde. Secretaria de Vigilância em Saúde. Vigilância em saúde no SUS: fortalecendo a capacidade de resposta aos velhos e novos desafios. Brasília (DF); 2006. (Série B. Textos Básicos de Saúde).

27. Moysés SJ, Pucca Junior GA, Paludetto Junior M, Moura L. Avanços e desafios à Política de Vigilância à Saúde Bucal no Brasil. Rev Saúde Pública. 2013;47(Suppl.3):161-7. http://dx.doi. org/10.1590/S0034-8910.2013047004329.

28. Brasil. Ministério da Saúde. Secretaria Nacional de Vigilância Sanitária. Portaria $n^{0} 22$, de 20 de dezembro de 1989. Diário Oficial União. 22 dez 1989; Seção II:241.

29. Brasil. Ministério da Saúde. Secretaria Nacional de Vigilância Sanitária. Portaria $n^{\circ} 108$, de 26 de setembro de 1994. Diário Oficial União. 28 set 1994; p.14704.

30. Brasil. Ministério da Saúde. Secretaria Nacional de Vigilância Sanitária. Portaria $n^{\circ} 71$, de 29 de maio de 1996. Aprova a relação de documentos necessários à formação de processos para autorização, alteração e cancelamento de funcionamento de empresa, registro de produto, suas alterações, revalidação, cancelamento e outros procedimentos afins, conforme anexos I, II, III, IV, V, VI, VII, VIII, IX e X e dá outras providências. Diário Oficial União. 4 jun 1996; p.9821-3. 
31. Cury JA, Tenuta LMA, Ribeiro CCC, Paes Leme AF. The importance of fluoride dentifrices to the current dental caries prevalence in Brazil. Braz Dent J. 2004;15(3):167-74. doi:10.1590/ S0103-64402004000300001

32. Matias JB, Azevedo CS, do Vale HF, Rebelo MAB, Cohen-Carneiro F. Fluoride stability in dentifrices stored in schools in a town of northern Brazil. Braz Oral Res. 2015;29(1):S180683242015000100304. doi: 10.1590/1807-3107BOR-2015.vol29.0121.

33. Hashizume LN, Lima YBO, Kawaguchi Y, Cury JA. Fluoride availability and stability of Japanese dentifrices. J Oral Sci. 2003 Dec;45(4):193-9.

34. Cury JA, Tabchoury CPM, Piovano S. Concentration and stability of fluoride in dentifrices market in Buenos Aires. Bol Asociac Argent Odontol Niños. 2006 Jun/Sep;35(2):4-8.

35. Jordan RA, Markovich L, Gaengler P, Zimmer S. Total and free fluoride concentrations of African dentifrices marketed in West Africa. Oral Health Prev Dent. 2011;9(1):53-8. doi:10.3290/j. ohpd.a21285

36. Benzian H, Holmgren C, Buijs M, Loveren C, Weijden F, Palenstein Helderman W. Total and free available fluoride in toothpastes in Brunei, Cambodia, Laos, the Netherlands and Suriname. Int Dent J. 2012;62(4):213-21. doi:10.1111/j.1875-595X.2012.00116.x

37. Carrera CA, Giacaman RA, Muñoz-Sandoval C, Cury JA. Total and soluble fluoride content in commercial dentifrices in Chile. Acta Odontol Scand.2012;70(6):583-8. doi:10.3109/00016357 .2011 .640287

38. Chávez BA, Vergel GB, Cáceres CP, Perazzo MF, Vieira-Andrade RG, Cury JA. Fluoride content in children's dentifrices marketed in Lima, Peru. Braz Oral Res. 2019 Jul;33:e051. doi: 10.1590/1807-3107bor-2019.vol33.0051.

39. European Union. Statutory Instruments. Consumer Protection: The Cosmetic Products (Safety) Regulations 2008: $\mathrm{N}^{\mathrm{o}}$ 1284. London: Stationery Office; 2008 [citado 2019 set 30]. Disponível em: http://www.legislation.gov.uk/uksi/2008/1284/ pdfs/uksi_20081284_en.pdf

40. MERCOSUL. MERCOSUL/GMC/RES No 48/02. Regulamento técnico MERCOSUL sobre lista de substâncias que os produtos de higiene pessoal, cosméticos e perfumes não devem conter, exceto nas condições e com as restrições estabelecidas. Brasília (DF): Sistema de Informação do Comércio Exterior; 2002 [citado 2019 set 30]. Disponível em: http:/www.sice.oas.org/ 
trade/mrcsrs/resolutions/res4802p.asp

41. US Food and Drug Administration. CFR - Code of Federal Regulations. Title 21: Food and drugs. Washington (DC); 2013 [citado 2019 set 30]. Chapter I, Food and Drug Administration Department of Health and Human Services, Subchapter D, Drugs for human use (part 355): anticaries drugs products for over the counter human use. Disponível em: http://www.gpo.gov/ fdsys $/$ browse $/$ I\&oldPath=Title $+21 \&$ isCollapsed=true\&selectedYearFrom $=2013^{\circ}$ ycord=710.

42. Bureau des normes de Madagascar. Medecine bucco-dentaire - dentifrices - Exigences, methodes d'essai et marquage. [citado 2019 set 30]. Disponível em: http://blog.aoi-fr.org/wpcontent/uploads/2018/11/Normes-Dentifrice-Madagascra-aout-2018.pdf.

43. Do LG, Ha DH, Spencer AJ. Natural history and long-term impact of dental fluorosis: a prospective cohort study. Med J Aust. 2016 Jan 18;204(1):25. doi: 10.5694/mja15.00703.

44. Onoriobe U, Rozier RG, Cantrell J, King RS. Effects of enamel fluorosis and dental caries on quality of life. J Dent Res. 2014 Oct;93(10):972-9. doi:10.1177/0022034514548705.

Artigo apresentado em outubro de 2019 Artigo aprovado em janeiro de 2020 Artigo publicado em maio de 2020 